\section{Las primeras médicas de Chile y Latinoamérica: Eloísa Díaz Insunza y Ernestina Pérez Barahona}

\author{
CARLOS G. OSORIO ABARZÚA ${ }^{1}$
}

\section{The first chilean female doctors: Eloísa Díaz Insunza and Ernestina Pérez Barahona}

\begin{abstract}
Dr. Eloisa Díaz Insunza (1866-1950) was the first woman to become a doctor-surgeon in Chile and Latin America in 1887. Less known is her distinguished colleague, Dr. Ernestina Pérez Barahona (1865-1951), the second woman graduated in Chile as a physician, only seven days after Dr. Diaz. Dr. Diaz entered the School of Medicine of the University of Chile in 1881 and Dr. Pérez in 1883. However, both graduated from Bachelor of Medicine and Pharmacy in 1885 and received their degree in 1887. This paper highlights the extraordinary parallelism in their medical studies.
\end{abstract}

(Rev Med Chile 2019; 147: 367-371)

Key words: Chile; Health education; History of Medicine; Physicians, Women.

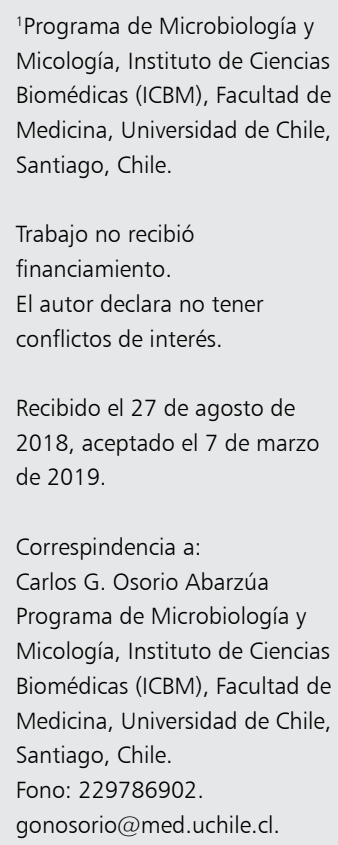

$\mathrm{P}$ ara apreciar la magnitud del esfuerzo de estas dos mujeres tenemos que retrotraernos a lo que era Chile y el mundo en el año 1887. En ese año sólo en dos países del mundo había mujeres médicas, estos eran Estados Unidos de Norteamérica e Inglaterra. En Estados Unidos de Norteamérica la primera mujer graduada en medicina fue Elizabeth Blackwell en 1849 a la edad de 28 años en el Geneva Medical College de Nueva York $^{1}$. En Inglaterra, Elisabeth Garrett Anderson obtuvo su licencia para practicar la medicina en $1865^{2}$. Elena Caffarena Morice en un discurso en honor a Ernestina Pérez en 1953 dice lo siguiente ${ }^{3}$ : "Ubiquémonos ahora en el Chile de 1887, con menos de un siglo de independencia política, aplastado con prejuicios religiosos y arrastrando el tremendo lastre feudal de la herencia española. Las mujeres vivían agobiadas, no sólo por el peso de las costumbres coloniales, sino que hasta físicamente por ropas que las cubrían de la cabeza a los pies. Cuando Ernestina Pérez recibió su título de médica, no existía en Chile ningún colegio femenino de Enseñanza Secundaria del Estado, ya que el primer Liceo Fiscal de Niñas, se fundó en Valparaíso en 1891".

La primera mujer en criticar el lamentable estado de la educación nacional femenina en dicha época fue doña Antonia Tarragó González, quien había fundado en 1864 el establecimiento privado Colegio Santa Teresa para alumnas secundarias en Santiago. En octubre de 1872, doña Antonia solicitó al Consejo Universitario que se permitiera a sus alumnas egresadas rendir las pruebas de ingreso al sistema universitario. La iniciativa no tuvo éxito. Cuatro años después, en diciembre de 1876, el Consejo Universitario recibió una nueva solicitud de Isabel Le Brun Reyes, directora del establecimiento privado femenino Colegio de la Recoleta, en que pedía que las alumnas de su establecimiento pudieran rendir exámenes ante las comisiones universitarias ${ }^{4}$.

Finalmente, el 5 de febrero de 1877, el Ministro de Instrucción Pública señor Miguel Luis Amunátegui Aldunate, durante el gobierno del presidente Aníbal Pinto Garmendía (1876-1881), firmó el histórico y revolucionario decreto que 
permitió a las mujeres egresadas de la enseñanza media postular a los exámenes de admisión de la Universidad, cambiando para siempre el escenario de la instrucción pública chilena ${ }^{4}$.

Esta ley, en su parte más destacada, expresaba lo siguiente:

"Se declara que las mujeres deben ser admitidas a rendir exámenes válidos para obtener títulos profesionales, con tal que se sometan para ello a las mismas disposiciones a que están sujetos los hombres".

Finalmente se materializaba el sueño de las pioneras Antonia Tarragó e Isabel Le Brun. Tan sólo cuatro años después, en 1881, ingresaba a la Universidad de Chile la primera estudiante mujer. El cambio sustantivo de la sociedad ya era inevitable.

\section{Eloísa Díaz Insunza (1866-1950)}

Gran parte de los datos biográficos han sido extraídos del famoso Diccionario Biográfico de Chile de Pedro Pablo Figueroa ${ }^{5}$. Eloísa Díaz nació el 25 de junio de 1866 en Santiago de Chile (Figura 1). Sus padres fueron don Eulogio Díaz Varas y doña Carmela Insunza. Sus estudios básicos los realizó en el colegio de primeras letras que dirigía Dolores Cabrera Martínez. Las humanidades o enseñanza media las cursó en el colegio fundado y dirigido por Isabel Le Brun de Pinochet y en el Instituto Nacional ${ }^{5,6}$. El día lunes 11 de abril de 1881, antes de cumplir sus 15 años, obtuvo su grado de Bachiller en Filosofía y Humanidades. El diario El Ferrocarril publicó la siguiente noticia:

"El claustro universitario presentaba anoche

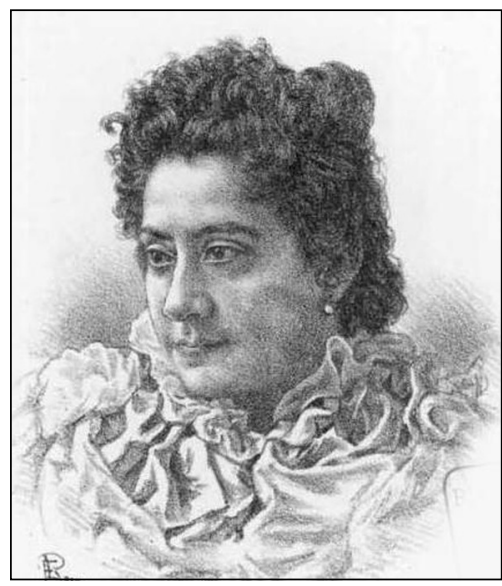

Figura 1. Retrato de la doctora Eloísa Díaz Insunza ${ }^{5}$. una animación que no es frecuente en ese augusto recinto de la ciencia. Por primera vez en Chile, figuraba entre las aspirantes al bachillerato en humanidades, un estudiante del sexo femenino, y tanto la novedad del hecho como la curiosidad despertada entre los alumnos de la sección universitaria, habían logrado atraer una numerosa concurrencia a la sala de examen. Las cédula sorteada comprendia la Historia de América y de Chile, sobre cuyos ramos sufrió el candidato un detenido examen. Inútil es decir que contestó satisfactoriamente a todas las preguntas y que manifestó un perfecto conocimiento de los sucesos que se han desarrollado en este continente y en especial en nuestro país. Su fácil expedición en todas las cuestiones le valió la aprobación unánime de la mesa, y el nombre del examinando, la señorita Eloisa Díaz Inzurza, fué vivamente aclamado por la concurrencia. Anoche mismo pasó el espediente al Consejo de Instrucción Pública y se le confirió el grado de bachiller en la facultad de filosofía y humanidades. El señor rector, al entregarle el diploma, le dirijió algunas palabras de felicitación, y por segunda vez la concurrencia prorrumpió en aplausos, aplausos que se renovaron al salir de la sala el nuevo bachiller".

\section{(diario El Ferrocarril de Santiago,} 12 de abril de 1881)

Unos días después, el 21 de abril de 1881, la joven Eloísa se reunió con el decano de la Escuela de Medicina de la Universidad de Chile, el doctor Adolfo Murillo Sotomayor, para que la incorporara a esa carrera. Se puede leer en el Mercurio de Valparaíso lo siguiente:

"La señorita bachiller en filosofía y humanidades doña Eloisa Díaz Insurza se presentó hoy al señor decano de medicina señor Murillo y solicitó su incorporación entre los alumnos del primer curso de medicina. El señor decano le manifestó su complacencia por ser la primera de su sexo que deseaba seguir la profesión médica; le dijo que no había inconveniente para permitirle estudiar con los demás alumnos los ramos de esa carrera, y le prometió recomendarla a los profesores y alumnos de las cátedras de medicina para que le guarden todas las consideraciones y el respeto debidos al sexo de la aspirante".

(El Mercurio de Valparaíso, 22 de abril de 1881). 
Luego de cuatro años de estudios, el 12 de enero de 1885, obtuvo su grado de Bachiller en Medicina y Farmacia. Se debe recordar que en esa época (desde 1860) los estudios médicos duraban en total seis años ${ }^{7}$. Después de cursar los primeros cuatro años los estudiantes recibían el grado de Bachiller en Medicina y Farmacia y finalmente al término de los seis años de estudios médicos se presentaba la memoria que permitía acceder al grado de Licenciado en Medicina y Farmacia, el que a su vez permitía obtener el título profesional de Médico Cirujano. La memoria de Eloísa Díaz para optar al grado de Licenciado se tituló: "Breves observaciones sobre la aparición de la pubertad en la mujer chilena y las predisposiciones patológicas del sexo". Esta memoria fue presentada el 25 de diciembre de 1886 obteniendo el grado de Licenciado en Medicina sólo dos días después, el 27 de diciembre de 1886. Finalmente obtuvo su título profesional el 3 de enero de 1887, cuando tenía tan sólo 20 años ${ }^{8}$.

\section{Ernestina Pérez Barahona (1865-1951)}

Existe una escasa cantidad de datos biográficos sobre Ernestina Pérez. Una razón importante para ello es que no tuvo el honor de aparecer en el famoso Diccionario Biográfico de Pedro Pablo Figueroa ${ }^{5}$. Ernestina nació el 8 de agosto de 1865 en Valparaíso (Figura 2) ${ }^{9}$. La enseñanza secundaria la realizó en el Liceo de Isabel Le Brun Reyes, al igual que Eloísa Díaz Insunza (Museo Nacional

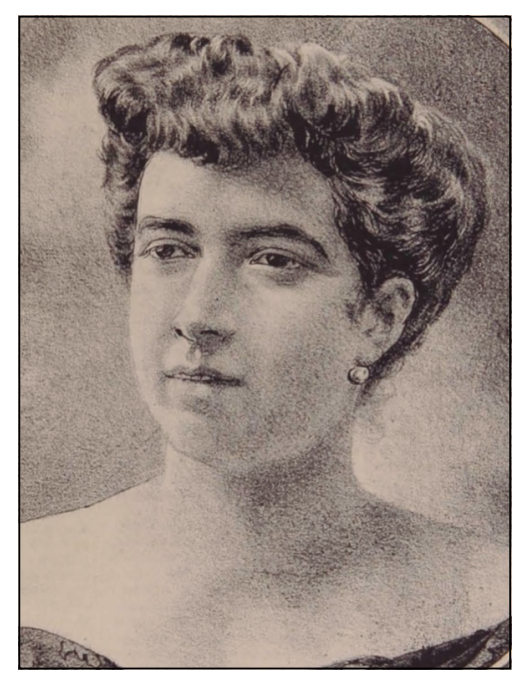

Figura 2. Retrato de la doctora Ernestina Pérez Barahona $^{12}$. de Medicina). Se graduó de Bachiller en Filosofía y Humanidades con fecha 8 de enero de 1883 , ingresando ese mismo día a la carrera de Medicina en la Universidad de Chile. Obtuvo su grado de Bachiller en Medicina y Farmacia el 14 de enero de 1885. Con fecha 31 de diciembre de 1886 recibió su grado de Licenciado en Medicina y Farmacia con su memoria titulada "Higiene social". El día 10 de enero de 1887, se recibió de médica de la Facultad de Medicina de la Universidad de Chile cuando tenía 21 años ${ }^{8}$. Es destacable, que antes de obtener su título profesional hubiera publicado dos artículos en la Revista Médica de Chile ${ }^{10,11}$.

\section{Paralelismos notables}

Nacieron y murieron con una diferencia de menos de un año (junio 1866-agosto 1865 y noviembre 1950-junio 1951, respectivamente). Ambas doctoras hicieron sus estudios secundarios en el prestigioso colegio privado de la pionera en educación femenina doña Isabel Le Brun Reyes (Figura 3). Este establecimiento fue fundado por doña Isabel Le Brun en 1875 con el nombre de Colegio de la Recoleta. Probablemente en sus inicios este establecimiento estaba ubicado en la entrada sur de calle Recoleta en el barrio de la Chimba, pero posteriormente fue trasladado a calle Duarte No 130 muy cerca de la Alameda (hoy calle Lord Cochrane).

Ingresaron a la Escuela de Medicina con una diferencia de casi dos años (1881 y 1883). Sin embargo, la doctora Pérez pudo de manera excepcional alcanzar a la doctora Díaz, obteniendo ambas el grado de Bachiller en Medicina y Farmacia en enero de 1885 con una diferencia de tan sólo dos días (Tabla 1). La expectación y sorpresa

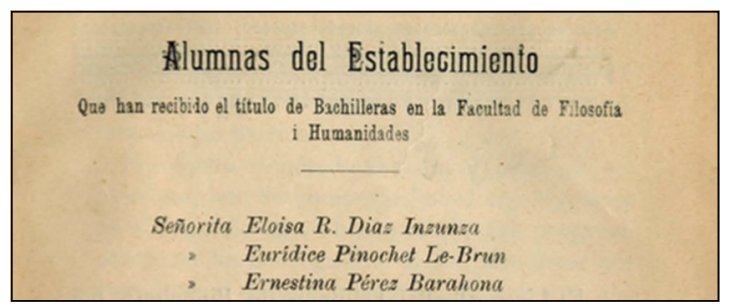

Figura 3. Primera página del prospecto del Liceo de Isabel Le Brun de Pinochet de 1900. Muestra a las tres primeras alumnas del Liceo que obtuvieron su grado de Bachiller en Filosofía y Humanidades ${ }^{13}$. 
Tabla 1. Fechas de obtención de grados académicos y títulos profesionales de Eloísa Díaz Insunza y Ernestina Pérez Barahona ${ }^{8}$

\begin{tabular}{|lll|}
\hline & Eloísa Díaz Insunza & Ernestina Pérez Barahona \\
\hline Bachiller en Filosofía y Humanidades & 11 abril de 1881 & 8 de enero 1883 \\
\hline Bachiller en Medicina y Farmacia & 12 enero 1885 & 14 de enero 1885 \\
\hline Licenciado en Medicina y Farmacia & 24 de diciembre de 1886 & 31 de diciembre 1886 \\
\hline Título Médico Cirujano & 3 de enero 1887 & 10 de enero 1887 \\
\hline
\end{tabular}

de las autoridades universitarias ante estas dos destacadas mujeres cursando simultáneamente la carrera de medicina debe haber sido grande, lo que se percibe claramente en el grado de Bachiller en Medicina y Farmacia otorgado a Eloísa Díaz con fecha 12 de enero de 1885. El Boletín de Instrucción Pública de dicha fecha dice textualmente lo siguiente: "el señor rector confirió el grado... de Bachiller en Medicina i Farmacia a Ernestina Díaz Insunza, que ha sido la primera mujer que ha obtenido este grado en la Universidad de Chile"!! Tan grande era la sorpresa del cuerpo académico que una nueva alumna quimérica fue creada en la confusión. Para alcanzar a Eloísa, el esfuerzo de Ernestina debió ser indudablemente enorme, pues tuvo que comprimir 4 años de estudios en tan sólo 2. Es probable que sea un caso único en la carrera de Medicina de la Universidad de Chile, pero falta evidencia para confirmar este hecho. Posteriormente, la obtención del grado de Licenciatura en Medicina y Farmacia y la obtención del título de Médico-Cirujano ocurrió con tan sólo unos días de diferencia.

\section{Epílogo}

En su homenaje de 1953 Elena Caffarena comentó lo siguiente:

"Quise saber el por qué de esta diferencia de días entre un examen y otro e interrogué directamente sobre esto a la doctora Pérez. Ya que ambas han fallecido y pertenecen a la historia, creo no faltar a la discreción al repetir lo que Ernestina Pérez me dijo. Ambas amigas habían acordado dar juntas sus exámenes y que, por razones ignoradas, el expediente de la futura doctora Díaz fue colocado en una sesión de la facultad anterior a la suya. En honor a la verdad, debo recalcar que la doctora Pérez no insinuó cargo alguno contra su compañera de estudios, pero había en su rostro una expresión de malicia y viveza más acentuada que la corriente" 3 .

La doctora Ernestina Pérez insinuó, con cierta malicia y viveza, razones desconocidas para el adelantamiento del expediente de su compañera a una sesión de Facultad anterior a la convenida. En mi opinión, es ciertamente posible y comprensible, que la doctora Pérez haya pensado así. Sin embargo, creo muy probable que las autoridades de la Facultad de Medicina autónomamente y ponderadamente hayan establecido una pequeña diferencia en las fechas de obtención de los distintos grados y título profesional de ambas alumnas con el fin de otorgarle a la doctora Díaz el honor de haber sido la primera mujer en Chile y Latinoamérica en ingresar a la carrera de Medicina. Esa mínima diferencia de siete días significó que Eloísa tuviera un lugar destacado en el Diccionario Biográfico de Chile, pero que Ernestina ni siquiera fuera mencionada. A la distancia histórica que nos encontramos hoy de los hechos narrados es muy difícil explicar la diferencia sistemática de pocos días en las fechas de los grados de Bachiller y Licenciado en Medicina y Farmacia y en el título de Médico-Cirujano. Las contingencias históricas se han esfumado en el tiempo y tan sólo es posible especular sobre los escenarios posibles. Hoy, sin la presión del momento histórico y con la enorme distancia de los años transcurridos, me atrevería a sugerir la aplicación de la famosa "navaja de Occam" (essentia non sunt multiplicanda praeter necessitatem; las esencias no deben multiplicarse más allá de lo necesario o en otras palabras: debemos quedarnos con la hipótesis más simple) para este caso, pero no en el sentido original de seleccionar la hipótesis más simple sino más bien de establecer la mirada más ecuánime. Mi visión, 
respecto a estas dos extraordinarias mujeres, es que ambas merecen con toda justicia ser consideradas por la posteridad como las primeras médicas de Chile y Latinoamérica.

Agradecimientos: Quisiera agradecer a varios colegas del Programa de Microbiología del Instituto de Ciencias Biomédicas (ICBM) que accedieron a leer y criticar trabajo, ayudanado a mejorar sustancialmente su presentación.

\section{Referencias}

1. Markel H. 2014. How Elizabeth Blackwell became the first female doctor in the US. Documento digital: https://deepblue.lib.umich.edu/bitstream/handle/2027.42/102531/ PBSNewsHour_Blackwell.pdf. (Consultado el 20 de julio de 2018).

2. Kelly L. Elizabeth Garrett Anderson: early pioneer of women in medicine. The Lancet 2017; 390 (10113): 2620-1.

3. Unión Chilena de Mujeres. 1953. Homenaje a la Dra. Ernestina Pérez Barahona. Disponible en sitio: http:// www.memoriachilena.cl/. (Consultado el 5 de julio de 2018).
4. Vera J. El sistema de admisión a la Universidad: permanencia y cambio. Editorial Universitaria. Santiago, Chile, 2015.

5. Figueroa PP. Diccionario Biográfico de Chile. 4 edición, Santiago, Chile, 1897. Disponible en sitio: http://www. memoriachilena.cl/. (Consultado el 20 de agosto de 2018).

6. Jímenez A. Dra. Eloísa Díaz Insunza. Rev Chilena Infectol 2000; 17 (1): 76-8.

7. Sierra L. Cien años de la enseñanza de la medicina en Chile. An Fac Biol Méd 1934; 1: 1-134.

8. Boletín de Instrucción Pública. Años 1881, 1885, 1886 y 1887. Disponibles en sitio web: https://anales.uchile.cl/. (Consultado el 3 de marzo de 2018).

9. Museo Nacional de Medicina. Dra Pérez Ernestina. Disponible en sitio: http://www.museomedicina.cl/. (Consultado el 15 de agosto de 2018).

10. Pérez E. Útero bicorne. Rev Med Chile (1885-1886); 14: 587-8.

11. Pérez E. Resección Tarsiana. Rev Méd Chile (18861887); 15: 188-9.

12. Revista Lira Chilena. Año VII, número 20, Santiago, Chile, 1904. Disponible en sitio: http://www.memoriachilena.cl/ (Consultado el 10 de agosto de 2018).

13. Prospecto Liceo Isabel de Brun de Pinochet. Santiago de Chile, 1900. Disponible en sitio: http://www.museodelaeducacion.cl/. Consultado el 3 de marzo de 2018. 